\title{
The Association Between Obesity and Social Isolation as Well as Loneliness in the Adult Population: A Systematic Review
}

\section{André Hajek (1D) \\ Benedikt Kretzler \\ Hans-Helmut König (iD}

Department of Health Economics and Health Services Research, University Medical Center Hamburg-Eppendorf, Hamburg, 20246, Germany
Correspondence: André Hajek Department of Health Economics and Health Services Research, University Medical Center Hamburg-Eppendorf, Martinistraße 52, Hamburg, 20246, Germany

Tel +4940741052877

Fax $+49407410-40261$

Email a.hajek@uke.de
Purpose: Obesity is associated with adverse health outcomes and can result in feelings of loneliness or social isolation, for example due to stigmatization. These factors are in turn associated with morbidity and mortality. Thus far, a systematic review is lacking with regard to the association between obesity, social isolation and loneliness. Therefore, our aim was to fill this gap in knowledge.

Materials and Methods: Three established electronic databases (PubMed, PsycInfo, as well as CINAHL) were searched. Observational studies examining the link between obesity and loneliness or social isolation were included. Disease-specific samples were excluded.

Results: In sum, six studies have been included. While some studies point to an association between obesity and increased loneliness levels, it should be acknowledged that findings are mixed. Study quality was quite high.

Conclusion: This systematic review showed that the literature is inconclusive and incomplete. Further research is required to shed further light on these associations. Moreover, studies from countries outside Europe, based on longitudinal data and using objective assessments of obesity, are urgently required. Gender differences should be further explored. Keywords: excess weight, loneliness, obesity, overweight, social exclusion, social isolation

\section{Introduction}

Obesity is known as an "abnormal or excessive fat accumulation that presents a risk to health". ${ }^{1}$ It is a severe risk factor for non-communicable diseases in high income countries, $^{2}$ for example cardiovascular diseases or diabetes mellitus. ${ }^{3-5}$ Furthermore, it has been shown that obesity is associated with an increased economic burden. ${ }^{6}$ Moreover, it is associated with discrimination, decreased mental health or increased loneliness. ${ }^{7-9}$ About $13 \%$ of adults in Europe are obese. ${ }^{10}$ For example, higher prevalence rates have been reported in the United States. ${ }^{11}$

Similarly, both loneliness (feeling that one's own social network is smaller than desired $^{12}$ ) and social isolation (emotion of not belonging to the society ${ }^{13}$ ) can have marked effects on morbidity and mortality. It should be noted that loneliness and social isolation are correlated, but do not measure the same phenomenon. Additional details are given elsewhere. ${ }^{14,15}$

To date, some studies have investigated the association between obesity and loneliness or social isolation, ${ }^{16-19}$ with somewhat mixed evidence. For example, a recent longitudinal study conducted in Germany showed that the onset of 
obesity is associated with increased loneliness scores in men, but not in women, ${ }^{16}$ whereas another longitudinal study from Germany found that the onset of obesity was associated with a decrease in loneliness in men, but with an increase in loneliness in women. ${ }^{17}$ Factors such as media pressure, social norms or stigmatization may explain a link between obesity and increased loneliness/ isolation scores particularly among women. ${ }^{20}$ The association between an onset of obesity and decreased loneliness in (older) men ${ }^{17}$ may be explained by the fact that they feel accepted in society. They might be perceived as sociable. Similarly, a cross-sectional association between obesity and decreased social isolation among older women $^{21}$ may be explained by these factors, ie older women with obesity may feel accepted by friends and relatives. $^{21}$ Hansson and Rasmussen also showed that higher age is associated with more positive views about obesity. $^{22}$ In contrast, stigmatization is particularly present in children with obesity ${ }^{23}$ or young adults. ${ }^{24}$

Thus far, a systematic review is lacking systematically synthesizing evidence regarding these associations. Therefore, the aim of this current systematic review is to fill this gap in knowledge. In sum, this knowledge may assist in maintaining social and physical health during lifetime.

\section{Materials and Methods}

The methods of this review are in accordance with the Preferred Reporting Items for Systematic Reviews and Meta-Analysis (PRISMA) guidelines. ${ }^{25}$ Our work was registered to the International Prospective Register of Systematic Reviews (PROSPERO, registration number: CRD42021235625).

\section{Search Strategy and Selection Criteria}

In February/March 2021, a systematic literature search was performed in three electronic databases (PubMed, PsycINFO, CINAHL). In Table 1, the search query for PubMed is shown. Based on our own past studies in this research area and common terms in these areas, our search query was developed.

Two reviewers (AH, BK) independently assessed studies for inclusion (1. title/abstract screening; 2. full-text screening). Furthermore, a hand search (manually) was performed using the references of the eligible articles and using forwards citation tracking. Discussions were used in case of disagreement. This procedure was also used when disagreements occurred in extracting the data and assessing the quality of the studies.
Table I Search Strategy (PubMed)

\begin{tabular}{|l|l|}
\hline$\#$ & Search Term \\
\hline$\# 1$ & Excess weight \\
\hline$\# 2$ & Obes* \\
\hline$\# 3$ & Adipos* \\
\hline$\# 4$ & $\# I$ OR \#2 OR \#3 \\
\hline$\# 5$ & Loneliness \\
\hline$\# 6$ & Social exclusion \\
\hline$\# 7$ & Social isolation \\
\hline$\# 8$ & \#5 OR \#6 OR \#7 \\
\hline$\# 9$ & \#4 AND \#8 \\
\hline
\end{tabular}

Notes: The number sign (\#) refers to the search order. The asterisk $\left(^{*}\right)$ is a truncation symbol.

Inclusion criteria for our current review were: (1) Cross-sectional and longitudinal observational studies investigating the link between obesity and loneliness or social isolation. (2) Studies adequately assessing these constructs. (3) Studies published in peer-reviewed journals (German or English language). In contrast, studies were excluded if they solely analyzed samples with specific disorders such as participants with systemic diseases. No restrictions were applied regarding demographic factors, location or time. This also means that studies investigating children and adults would be included. Thus, no restrictions regarding the age have been applied.

We performed a pretest prior to our final eligibility criteria (with a sample of 100 titles/abstracts). However, our eligibility criteria were not adjusted.

\section{Data Extraction and Analysis}

One reviewer $(\mathrm{BK})$ performed the extraction of the data. A second reviewer $(\mathrm{AH})$ cross-checked it. If needed, the study authors were contacted for clarification. The extraction of the data included study design, assessment of obesity and loneliness/social isolation, sample characteristics, and key findings. The key results will be presented as follows (in each case: (1) cross-sectional, (2) longitudinal):

(1) Obesity and loneliness

(2) Obesity and social isolation

\section{Quality Assessment}

The well-known and widely used NIH Quality Assessment Tool for Observational Cohort and Cross-Sectional Studies ${ }^{26}$ 
was used to assess the study quality. Two reviewers (AH, BK) independently assessed the study quality.

\section{Results}

\section{Study Overview}

A flow chart is given in Figure $1 .{ }^{25}$ In total, $n=6$ studies were included in the final synthesis of our current systematic review. ${ }^{8,16-19,21}$ Key characteristics and main findings are displayed in Table 2. Adjusted results (if reported) are shown in Table 2.

Data exclusively stemmed from Europe ( $\mathrm{n}=6$, with four studies from Germany, ${ }^{8,16,17,21}$ and one study each from Finland ${ }^{19}$ and United Kingdom ${ }^{18}$ ). Equally, three crosssectional $^{18,19,21}$ and three longitudinal studies ${ }^{8,16,17}$ were found. In the longitudinal studies, the period of observation ranged from 20 months to 12 years.

The German studies used data from large cohort studies. ${ }^{8,16,17,21}$ Moreover, Sarlio-Lähteenkorva and Lahelma ${ }^{19}$ used data from the nationwide Finnish 'Survey of Living Conditions' (1994) which were collected by the government statistical authorities (Statistics Finland), whereas Rotenberg et $\mathrm{al}^{18}$ used data from undergraduates from a mid-size University in the United Kingdom. The sample size ranged from $137^{18}$ to $21,099 .{ }^{8}$ The proportion of women in the samples ranged from $45 \%{ }^{19}$ to $67.9 \%{ }^{16}$ and the mean age ranged from $22^{18}$ to 90 years. ${ }^{16}$ The average age was above 60 years in all four German studies. ${ }^{8,16,17,21}$ Additional details are presented in Table 2.

\section{Obesity and Loneliness}

Regarding cross-sectional studies, two studies examined the association between obesity and loneliness. ${ }^{18,19}$ While one study found an association between obesity and increased loneliness levels among undergraduates in the United Kingdom, ${ }^{18}$ the second study did not find an association between these factors in the general population in Finland. ${ }^{19}$ While the first study did not examine gender differences, the second study reported gender-stratified regression analyses. They did not find an association in both women and men. Gender differences were not tested using interaction terms.

With regard to longitudinal studies, two studies examined the association between obesity and loneliness in Germany. ${ }^{8,17}$ One study showed that the onset of obesity is associated with increased loneliness scores in men, but not in women (with significant gender differences). ${ }^{8}$ In contrast, a second study ${ }^{17}$ showed that the onset of obesity was associated with a decrease in loneliness in men, but with an increase in loneliness in women. The end of obesity was not associated with changes in loneliness scores (in both women and men). Gender differences were not tested using interaction terms.

\section{Obesity and Social Isolation}

With regard to cross-sectional studies, one study ${ }^{21}$ examined the association between obesity and (perceived) social isolation in Germany. This study found an association between obesity and decreased social isolation among women, but not in the total sample and in men. Gender differences were significant.

Regarding longitudinal studies, one study examined the association between obesity and (objective) social isolation in Germany. ${ }^{16}$ This study did not identify an association between obesity and social isolation among the oldest old.

\section{Study Quality}

The assessment of study quality of the eligible studies is displayed in Table 3. Most criteria were fulfilled by (nearly) all studies such as the adjustment of key covariates or the clear definition of the study population, whereas other factors were fulfilled by a few studies (eg, sufficient timeframe, response rate or attrition bias). Nevertheless, it should be noted that the general study quality was high: While five studies were rated as "good", 8,16,17,19,21 one study $^{18}$ was rated as "fair" and none of the studies were rated as "poor".

\section{Discussion \\ Main Findings}

In sum, six studies have been included in the final synthesis. While some studies point to an association between obesity and increased loneliness levels, it should be acknowledged that findings are mixed. Study quality was quite high.

Thus, due to the rather inconclusive evidence, it remains an open question for future research whether obesity is associated with increased or decreased loneliness/isolation in women and men. It should be clarified whether factors such as stigmatization or sociability are of particular importance for these associations in certain age groups. 


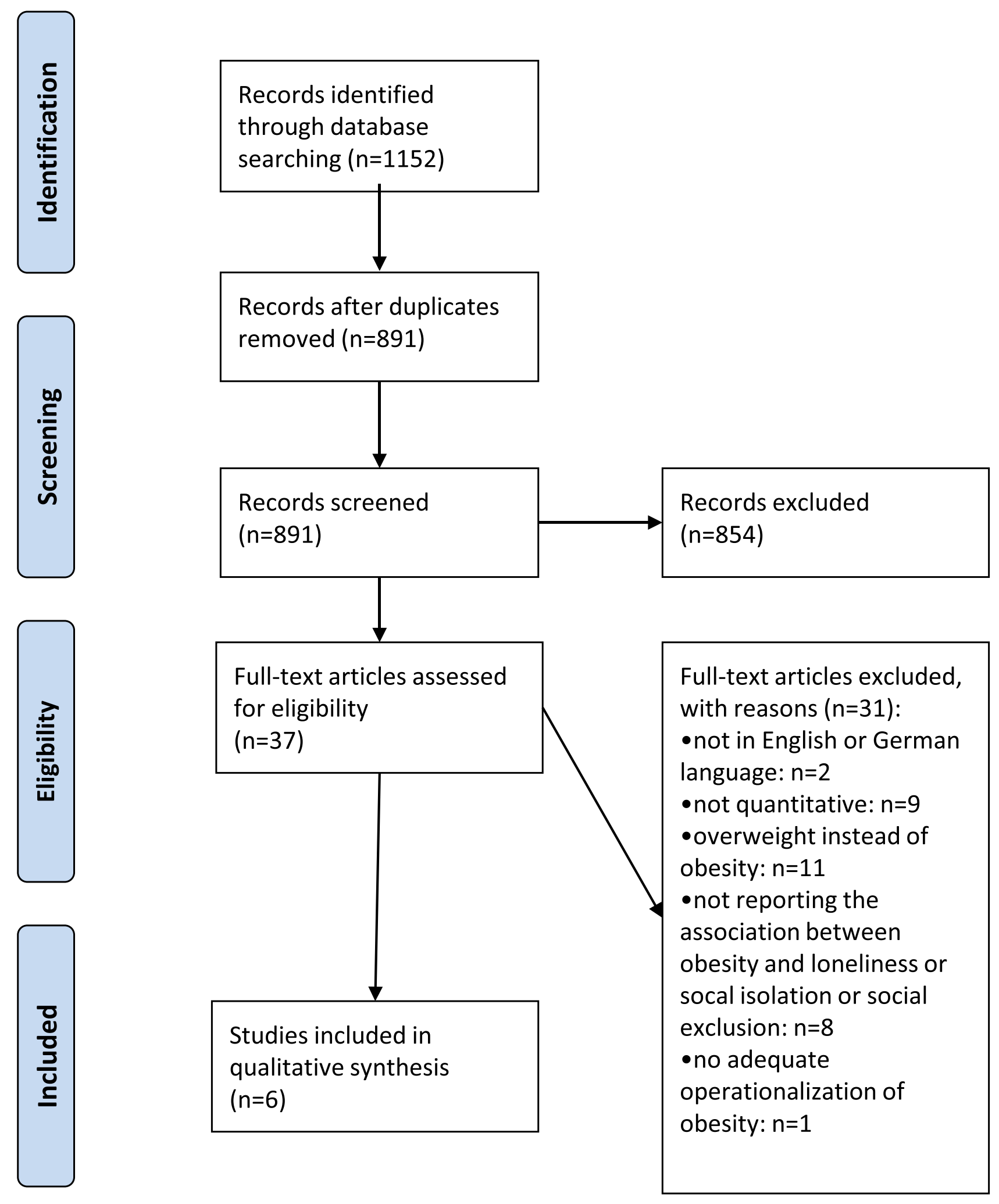

Figure I PRISMA flow chart.

Note: Adapted from Moher D, Liberati A, Tetzlaff J, Altman DG, Group P. Preferred reporting items for systematic reviews and meta-analyses: the PRISMA statement. PLoS Med. 2009;6(7):e1000097. ${ }^{25}$ 


\begin{tabular}{|c|c|c|c|c|c|c|c|}
\hline & 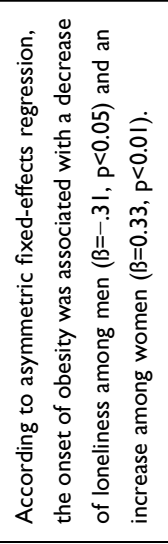 & 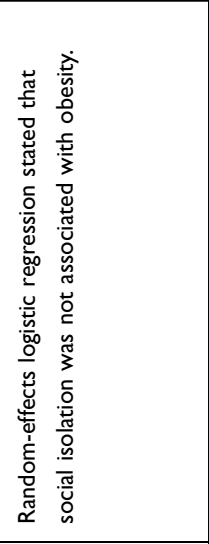 & 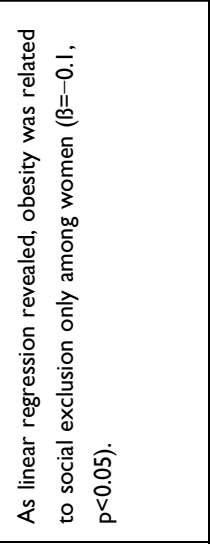 & 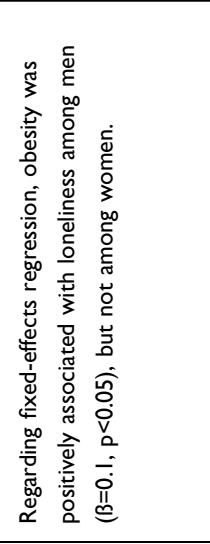 & 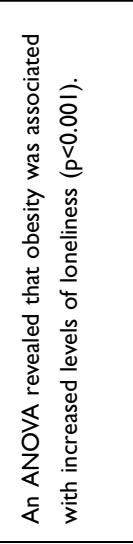 & 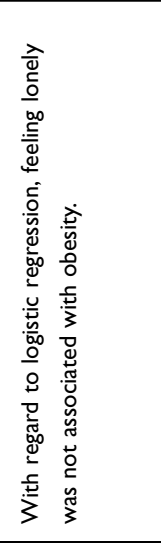 & \multirow[t]{2}{*}{ 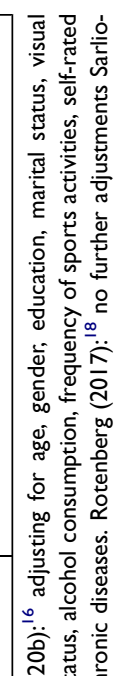 } \\
\hline 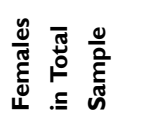 & $\frac{\stackrel{\circ}{i ்}}{\stackrel{\text { in }}{ }}$ & 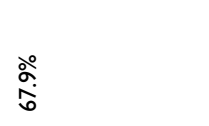 & $\frac{\circ}{\dot{\circ}}$ & ڤัণ & $\underset{\substack{\stackrel{\circ}{\circ} \\
\infty}}{\stackrel{\infty}{n}}$ & 号 & \\
\hline$\stackrel{8}{8}$ & 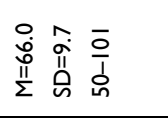 & 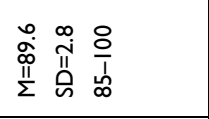 & 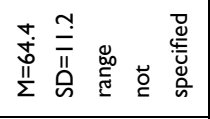 & 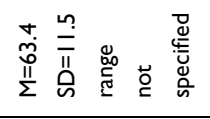 & 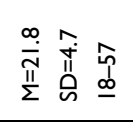 & 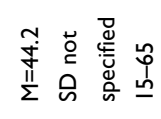 & 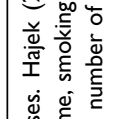 \\
\hline 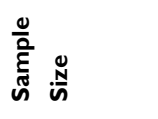 & $\begin{array}{l}\frac{j}{+} \\
\frac{0}{11} \\
=\end{array}$ & $\begin{array}{l}\stackrel{n}{0} \\
\text { II } \\
c\end{array}$ & $\underset{\substack{\infty \\
\prod}}{\stackrel{\infty}{\Sigma}}$ & $\begin{array}{l}\text { g. } \\
\stackrel{+}{\pi} \\
\text { II }\end{array}$ & $\frac{\hat{m}}{11}$ & $\begin{array}{l}\circ \\
\stackrel{0}{0} \\
\text { II } \\
\end{array}$ & 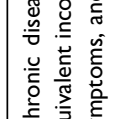 \\
\hline 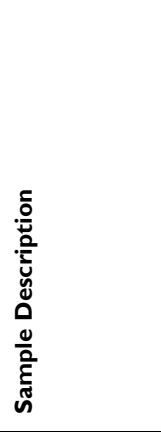 & 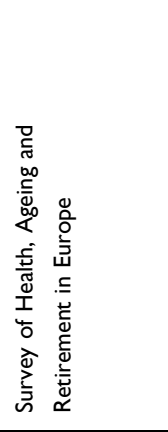 & 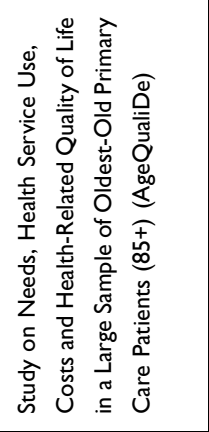 & 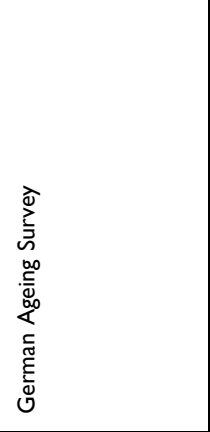 & 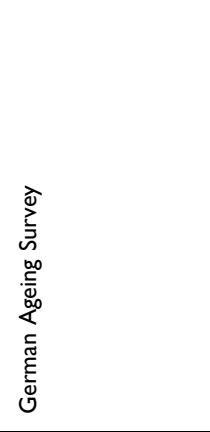 & 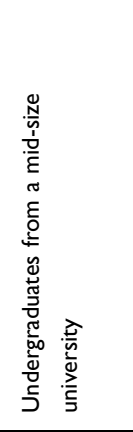 & 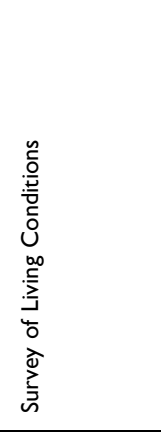 & 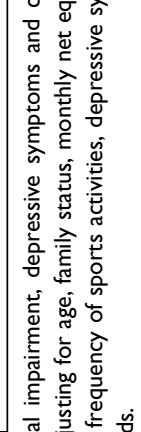 \\
\hline 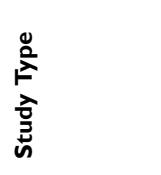 & 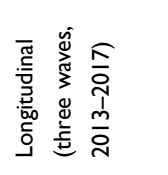 & 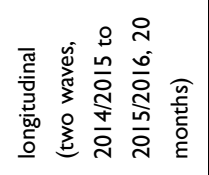 & 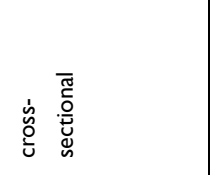 & 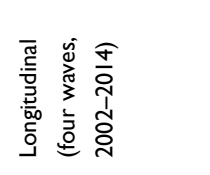 & 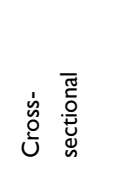 & 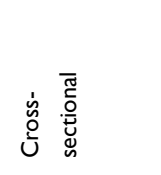 & 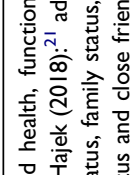 \\
\hline 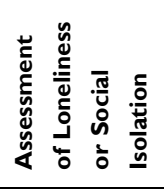 & 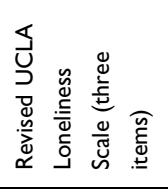 & 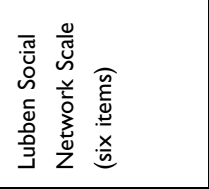 & 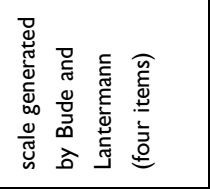 & 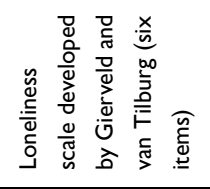 & 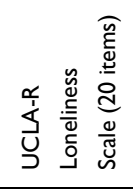 & 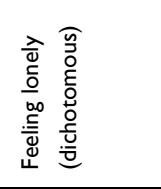 & 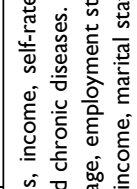 \\
\hline 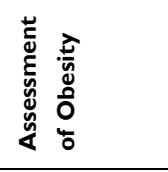 & 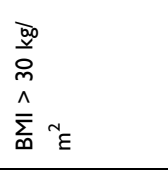 & $\begin{array}{l}\frac{\bar{s}}{0} \\
\dot{m} \\
\hat{\sum}_{\infty}^{N} E\end{array}$ & $\begin{array}{l}\frac{\bar{s}}{0} \\
\dot{m} \\
\hat{\sum_{\infty}}{ }^{N} E\end{array}$ & 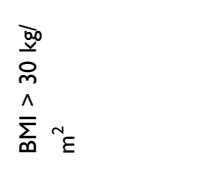 & $\begin{array}{l}\overline{\text { so }} \\
\overline{0} \\
\hat{\sum}_{\infty}{ }^{N} E\end{array}$ & $\begin{array}{l}\frac{\bar{s}}{0} \\
\hat{m} \\
\hat{\sum_{\infty}}{ }^{\prime} E\end{array}$ & 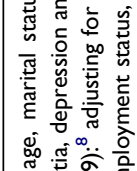 \\
\hline Uे & 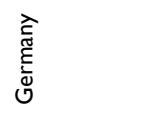 & 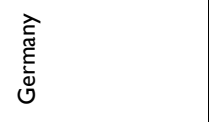 & 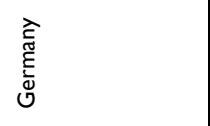 & 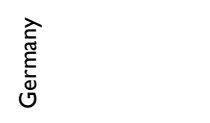 & 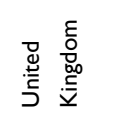 & 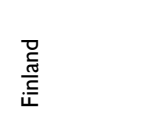 & 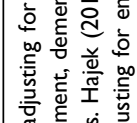 \\
\hline 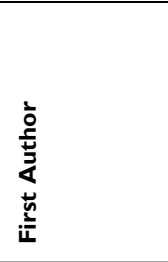 & 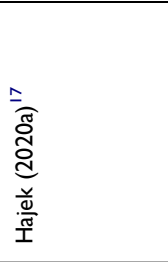 & 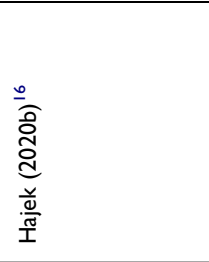 & 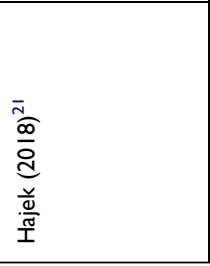 & 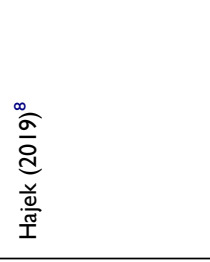 & 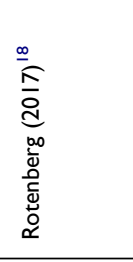 & 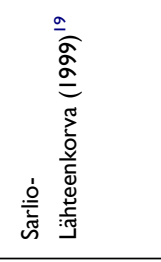 & 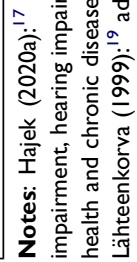 \\
\hline
\end{tabular}




\begin{tabular}{|c|c|c|c|c|c|c|c|c|c|c|c|c|c|c|}
\hline 总 & $\stackrel{\mathscr{\Perp}}{\check{\nu}}$ & $\stackrel{\mathscr{\nu}}{\check{\nu}}$ & 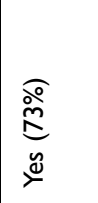 & $\stackrel{\tilde{y}}{\check{\nu}}$ & z & 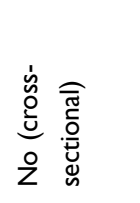 & 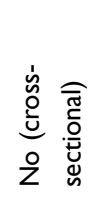 & 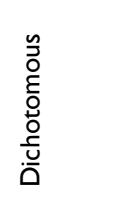 & $\stackrel{\tilde{\Perp}}{\check{\nu}}$ & \& & $\stackrel{\mathscr{\Perp}}{\check{\nu}}$ & 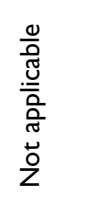 & $\stackrel{\mathscr{\varpi}}{\check{\nu}}$ & $\begin{array}{l}8 \\
8 \\
0\end{array}$ \\
\hline 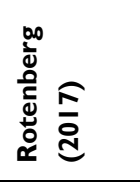 & $\stackrel{\Xi}{\check{\nu}}$ & $\stackrel{\mathscr{y}}{\check{\nu}}$ & 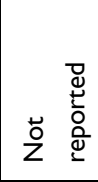 & $\stackrel{\tilde{\nu}}{=}$ & z & 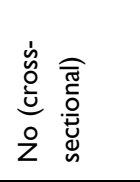 & 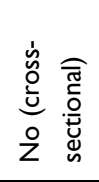 & 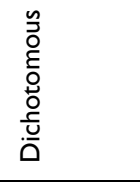 & $\stackrel{\tilde{y}}{\nu}$ & z & $\stackrel{\tilde{\nu}}{\check{\nu}}$ & 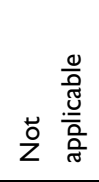 & z & 言 \\
\hline 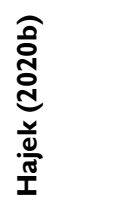 & $\stackrel{\mathscr{y}}{\check{\nu}}$ & $\check{\nu}^{\mathscr{y}}$ & 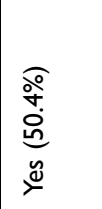 & $\stackrel{\mathscr{y}}{\nearrow}$ & $\stackrel{\circ}{z}$ & 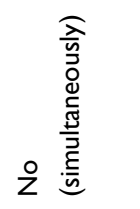 & $\stackrel{\tilde{\nu}}{\check{y}}$ & 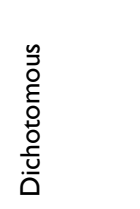 & $\stackrel{\tilde{y}}{\tau}$ & $\stackrel{\check{u}}{\sim}$ & $\stackrel{\tilde{\nu}}{\check{y}}$ & $\stackrel{\tilde{\nu}}{\tilde{y}}$ & $\stackrel{\tilde{\nu}}{\check{y}}$ & $\begin{array}{l} \\
\circ \\
0\end{array}$ \\
\hline 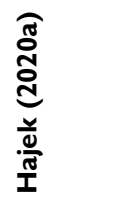 & $\stackrel{\mathscr{\varpi}}{\check{\nu}}$ & $\stackrel{\mathscr{\nu}}{\check{\nu}}$ & 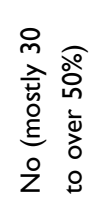 & $\stackrel{\tilde{\nu}}{\nu}$ & z & 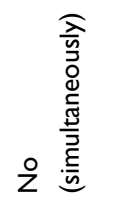 & $\stackrel{\check{\nu}}{\check{\nu}}$ & 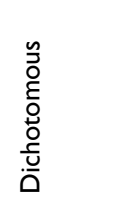 & $\stackrel{y}{\check{\nu}}$ & $\stackrel{\tilde{y}}{\check{0}}$ & $\stackrel{\mathscr{y}}{\check{\nu}}$ & $\stackrel{\mathscr{\nu}}{\check{\nu}}$ & $\stackrel{\mathscr{y}}{\check{\nu}}$ & $\begin{array}{l}0 \\
\circ \\
0\end{array}$ \\
\hline 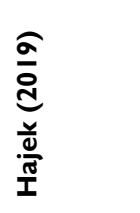 & $\stackrel{\mathscr{\Xi}}{\check{\nu}}$ & $\stackrel{\mathscr{\nu}}{\check{\nu}}$ & 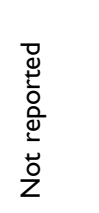 & $\stackrel{\tilde{\nu}}{\nu}$ & $\stackrel{\circ}{z}$ & 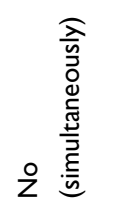 & $\stackrel{\tilde{\nu}}{\check{\nu}}$ & 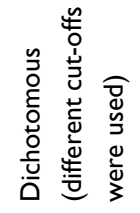 & $\stackrel{\tilde{y}}{\tau}$ & $\stackrel{\mathscr{u}}{\tau}$ & $\stackrel{\mathscr{y}}{\tau}$ & 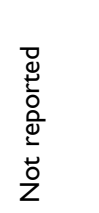 & $\stackrel{\tilde{y}}{\tau}$ & $\begin{array}{l}0 \\
8 \\
0 \\
0\end{array}$ \\
\hline 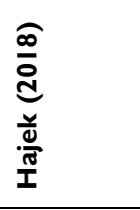 & 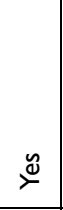 & $\stackrel{\check{\nu}}{\nu}$ & 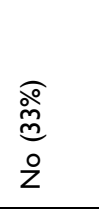 & 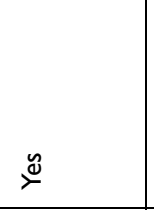 & z & 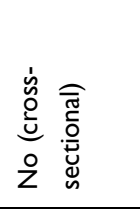 & 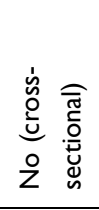 & 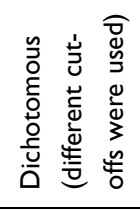 & $\stackrel{\check{\Xi}}{\check{0}}$ & z & $\stackrel{\mathscr{\Xi}}{\check{0}}$ & 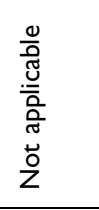 & $\stackrel{\tilde{u}}{\check{0}}$ & $\begin{array}{l}8 \\
8 \\
\end{array}$ \\
\hline 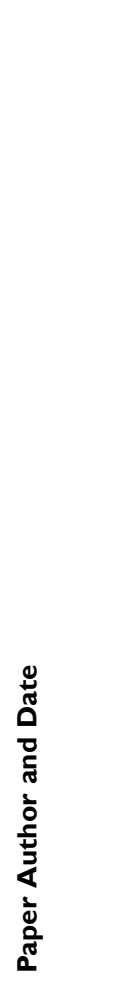 & 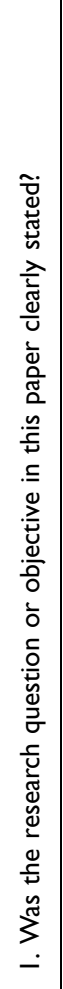 & 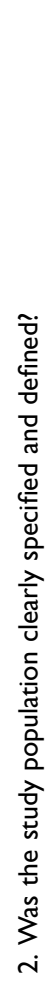 & 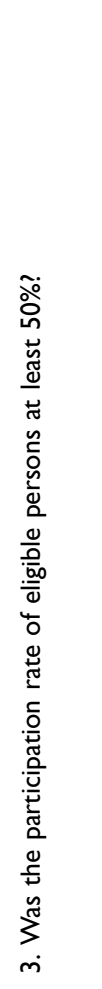 & 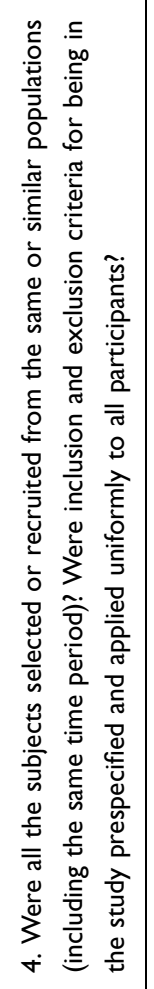 & 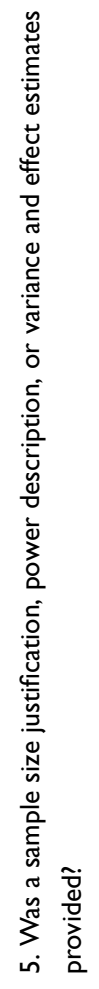 & 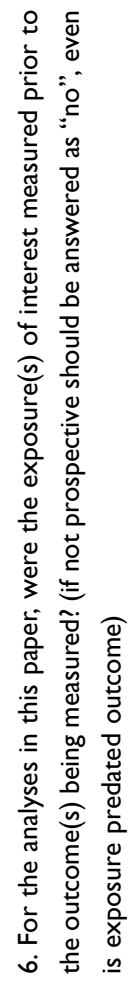 & 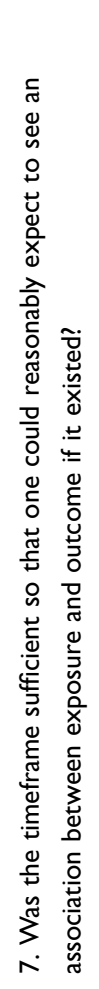 & 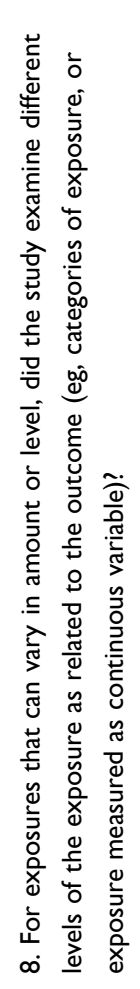 & 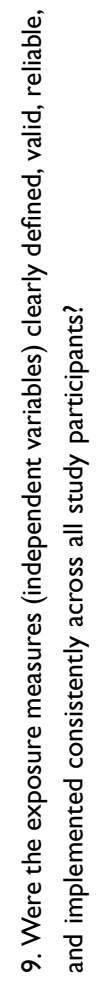 & 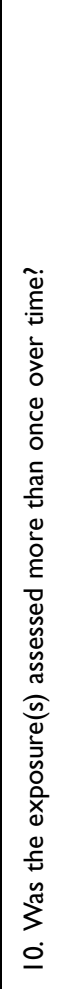 & 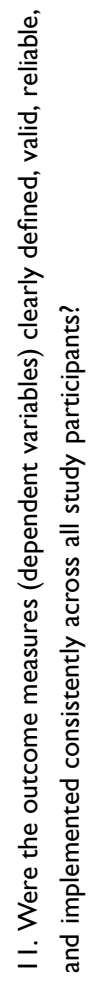 & 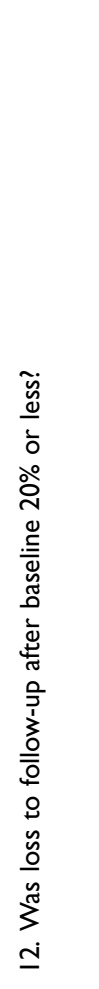 & 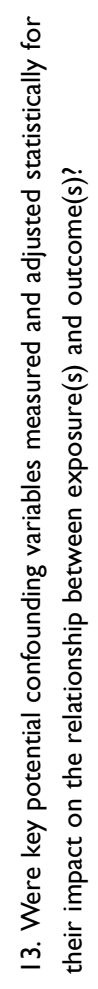 & 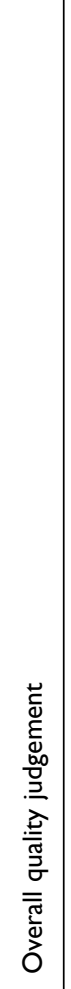 \\
\hline
\end{tabular}




\section{Comparability of the Included Studies}

Some factors limit the comparability of the studies included in our review. Self-rated BMI was used in all studies to quantify obesity, whereas different tools were used to quantify loneliness and social isolation. For example, while one study used a single item to quantify loneliness, ${ }^{19}$ a second study used the 20-item UCLA scale $^{27}$ and a third study used the De Jong Gierveld scale. ${ }^{28}$ Both tools (UCLA and de Jong Gierveld) treat loneliness as subjective. However, while the De Jong Gierveld tool mainly treats loneliness as cognitive, the UCLA scale mainly sees it as affective. ${ }^{29}$ Furthermore, the Lubben Social Network Scale covers objective social isolation, ${ }^{30}$ whereas the Bude and Lantermann tool ${ }^{13}$ covers perceived social isolation. These large differences in the tools used may partly explain the mixed findings.

Four out of the six samples used data from German samples focusing on older adults ${ }^{8,17,21}$ or oldest old. ${ }^{16}$ The other studies used samples from the general population (Finland) ${ }^{19}$ or a student sample ${ }^{19}$ - these differences may also explain the discrepancies in the findings. The longitudinal studies ${ }^{8,16,17}$ used appropriate panel regression models such as asymmetric fixed effects regressions ${ }^{31}$ which can produce consistent estimates. ${ }^{32}$

\section{Study Quality}

In total, there was a minor level of variety in the study quality. Beyond the factors described by the NIH tool, a common shortcoming was that it was sometimes not clarified how missing data were handled. Particularly in cross-sectional studies, this can lead to biased estimates. ${ }^{33}$ Strategies like full-information maximum likelihood $\left(\right.$ FIML) ${ }^{34}$ could be used in future studies. ${ }^{34}$ Contrary, in longitudinal studies, techniques like FIML often produce similar estimates (compared to default options like listwise deletion $^{35}$ ). The general study quality was quite high. This is rather unsurprising given the fact that five of the six studies were published in the past few years.

\section{Gaps in Knowledge and Guidance for Future Research}

Our current systematic review determined various gaps in knowledge. First, further longitudinal studies are needed to clarify the directionality between obesity and loneliness as well as social isolation. Second, studies from countries outside Europe are required. Third, the link between obesity and loneliness as well as social isolation during the times of the COVID-19 pandemic should be examined. For example, factors such as social network sites may play a key role during the pandemic. ${ }^{36}$ Fourth, since it is well known that self-reported BMI is prone to bias (since individuals tend to overestimate their height and underestimate their weight),${ }^{37}$ future studies based on objective assessments of obesity (eg, based on the hip to waist ratio or based on waist circumference) are required. Fifth, future studies examining gender differences are particularly important to understand the association between obesity and loneliness/isolation in further detail. Sixth and more generally, identifying potential moderating (eg, age group, educational level or personality characteristics such as extraversion) and mediating factors (eg, self-esteem or mental health) is important to gain a better understanding of the link between obesity and loneliness as well as social isolation.

\section{Strengths and Limitations}

Our current work is the first systematic review regarding the link between obesity and loneliness as well as social isolation. A quality assessment was performed. Two reviewers conducted key procedures such as selection of the studies, data extraction or assessing the study quality. We restricted our search to studies published in peer-reviewed journals since this may assure a certain study quality. Nevertheless, it should be acknowledged that some existing research (such as grey literature) may be excluded. Some studies may not be identified due to the language restrictions (our search was restricted to studies in German or English language). An electronic search in three databases has been done. However, two reviewers performed a hand search.

Furthermore, for reasons of study heterogeneity (eg, in the dependent variables used in the included studies), we did not conduct a meta-analysis - which is in line with the recommendations provided by Egger et al. ${ }^{38}$ According to Egger et al, performing a meta-analysis should be done with great caution when observational data are present because they can lead to incorrect estimates for various reasons. ${ }^{38}$

\section{Conclusion}

This systematic review showed that the literature is inconclusive and incomplete. Further research is required to shed further light on these associations since obesity is, among other things, associated with stigmatization. Moreover, studies from countries outside Europe, based on longitudinal data and using objective assessments of obesity are urgently required. Gender differences should be further explored. 


\section{Author Contributions}

$\mathrm{AH}, \mathrm{BK}$, and HHK, formulated the research question and designed the study; $\mathrm{AH}$ and $\mathrm{BK}$ conducted the literature search; AH and BK conducted the study selection, and data extraction processes; The manuscript was drafted by $\mathrm{AH}$ and critically revised by BK and HHK. All authors contributed to data analysis, drafting or revising the article, have agreed on the journal to which the article will be submitted, gave final approval of the version to be published, and agree to be accountable for all aspects of the work.

\section{Funding}

This research received no external funding.

\section{Disclosure}

The authors report no conflicts of interest in this work.

\section{References}

1. Organization WH. Obesity and overweight. Fact sheet no 311; 2015. http://www.who.int/mediacentre/factsheets/fs311/en/. Accessed June 4, 2021.

2. Finucane MM, Stevens GA, Cowan MJ, et al. National, regional, and global trends in body-mass index since 1980: systematic analysis of health examination surveys and epidemiological studies with 960 country-years and 9. 1 million participants. Lancet. 2011;377 (9765):557-567. doi:10.1016/S0140-6736(10)62037-5

3. Hall JE, Kuo JJ, da Silva AA, de Paula RB, Liu J, Tallam L. Obesityassociated hypertension and kidney disease. Curr Opin Nephrol Hypertens. 2003;12(2):195-200. doi:10.1097/00041552-20030300000011

4. Hernandez-Leon SG, Sarabia Sainz JA, Ramos-Clamont Montfort G, et al. Nanoproteomic Approach for Isolation and Identification of Potential Biomarkers in Human Urine from Adults with Normal Weight, Overweight and Obesity. Molecules. 2021;26(6):1803. doi:10.3390/molecules26061803

5. Lee J, Hwang Y, Kim K-N, et al. Associations of urinary sodium levels with overweight and central obesity in a population with a sodium intake. BMC Nutrition. 2018;4(1):1-14. doi:10.1186/ s40795-018-0255-6

6. König -H-H, Lehnert T, Brenner H, et al. Health service use and costs associated with excess weight in older adults in Germany. Age Ageing. 2015;44(4):616-623. doi:10.1093/ageing/afu120

7. Hajek A, König -H-H. Are changes in body-mass-index associated with changes in depressive symptoms? Findings of a population-based longitudinal study among older Germans. BMC Psychiatry. 2018;18(1):182. doi:10.1186/s12888-018-1748-1

8. Hajek A, König HH. Obesity and loneliness. Findings from a longitudinal population-based study in the second half of life in Germany. Psychogeriatrics. 2019;19(2):135-140. doi:10.1111/ psyg. 12375

9. Spahlholz J, Baer N, König HH, Riedel-Heller S, Luck-Sikorski C. Obesity and discrimination-a systematic review and meta-analysis of observational studies. Obesity Rev. 2016;17(1):43-55. doi:10.1111/ obr. 12343

10. Gallus S, Lugo A, Murisic B, Bosetti C, Boffetta P, La Vecchia C. Overweight and obesity in 16 European countries. Eur J Nutr. 2015;54(5):679-689. doi:10.1007/s00394-014-0746-4
11. Ogden CL, Carroll MD, Curtin LR, McDowell MA, Tabak CJ, Flegal KM. Prevalence of overweight and obesity in the United States, 1999-2004. JAMA. 2006;295(13):1549-1555. doi:10.1001/ jama.295.13.1549

12. Wenger GC, Davies R, Shahtahmasebi S, Scott A. Social isolation and loneliness in old age: review and model refinement. Ageing Soc. 1996;16(3):333-358. doi:10.1017/S0144686X00003457

13. Bude H, Lantermann E-D. Soziale exklusion und exklusionsempfinden. KZfSS Kölner Zeitschrift für Soziologie und Sozialpsychologie. 2006;58 (2):233-252. doi:10.1007/s11575-006-0054-1

14. Dahlberg L. Loneliness during the COVID-19 pandemic. Aging Ment Health. 2021;1-4.

15. Petersen N, König HH, Hajek A. The link between falls, social isolation and loneliness: a systematic review. Arch Gerontol Geriatr. 2020;88:104020. doi:10.1016/j.archger.2020.104020

16. Hajek A, Brettschneider C, van der Leeden C, et al. Prevalence and factors associated with obesity among the oldest old. Arch Gerontol Geriatr. 2020;89:104069. doi:10.1016/j.archger.2020.104069

17. Hajek A, König-H-H. Asymmetric effects of obesity on loneliness among older Germans. Longitudinal findings from the Survey of Health, Ageing and Retirement in Europe. Aging Ment Health. 2020;1-5.

18. Rotenberg KJ, Bharathi C, Davies H, Finch T. Obesity and the social withdrawal syndrome. Eat Behav. 2017;26:167-170. doi:10.1016/j. eatbeh.2017.03.006

19. Sarlio-Lähteenkorva S, Lahelma E. The association of body mass index with social and economic disadvantage in women and men. Int J Epidemiol. 1999;28(3):445-449. doi:10.1093/ije/28.3.445

20. Lennon SJ, Lillethun A, Buckland SS. Attitudes toward social comparison as a function of self-esteem: idealized appearance and body image. Family Consumer Sci Res J. 1999;27(4):379-405. doi:10.1177/1077727X99274001

21. Hajek A, König -H-H. The association between obesity and social exclusion in middle-aged and older adults: findings from a nationally representative study in Germany. BMC Geriatr. 2018;18(1):1-7. doi:10.1186/s12877-018-0946-5

22. Hansson LM, Rasmussen F. Attitudes towards obesity in the Swedish general population: the role of one's own body size, weight satisfaction, and controllability beliefs about obesity. Body Image. 2014;11 (1):43-50. doi:10.1016/j.bodyim.2013.10.004

23. Sikorski C, Luppa M, Brähler E, H-h K, Riedel-Heller SG. Obese children, adults and senior citizens in the eyes of the general public: results of a representative study on stigma and causation of obesity. PLoS One. 2012;7:10. doi:10.1371/journal.pone.0046924

24. Latner JD, Stunkard AJ, Wilson GT. Stigmatized students: age, sex, and ethnicity effects in the stigmatization of obesity. Obes Res. 2005;13(7):1226-1231. doi:10.1038/oby.2005.145

25. Moher D, Liberati A, Tetzlaff J, Altman DG, Group P. Preferred reporting items for systematic reviews and meta-analyses: the PRISMA statement. PLoS Med. 2009;6(7):e1000097. doi:10.1371/ journal.pmed.1000097

26. Health NIo, National Heart Lung, and Blood Institute. Study Quality Assessment Tools. National Heart Lung, and Blood Institute; 2018.

27. Russell DW. UCLA Loneliness Scale (Version 3): reliability, validity, and factor structure. J Pers Assess. 1996;66(1):20-40. doi:10.1207/ s15327752jpa6601_2

28. Gierveld JDJ, Tilburg TV. A 6-item scale for overall, emotional, and social loneliness: confirmatory tests on survey data. Res Aging. 2006;28(5):582-598. doi:10.1177/0164027506289723

29. Penning MJ, Liu G, Chou PHB. Measuring loneliness among middle-aged and older adults: the UCLA and de Jong Gierveld loneliness scales. Soc Indic Res. 2014;118(3):1147-1166. doi:10.1007/ s11205-013-0461-1

30. Lubben J, Blozik E, Gillmann G, et al. Performance of an abbreviated version of the Lubben Social Network Scale among three European community-dwelling older adult populations. Gerontologist. 2006;46 (4):503-513. doi:10.1093/geront/46.4.503 
31. Allison PD. Asymmetric fixed-effects models for panel data. Socius. 2019;5:2378023119826441. doi:10.1177/2378023119826441

32. Brüderl J, Ludwig V. Fixed-effects panel regression. In: Wolf C, editor. The Sage Handbook of Regression Analysis and Causal Inference. Los Angeles: SAGE; 2015:327-357.

33. Allison PD. Missing Data. Vol. 136. Sage publications; 2001.

34. Von Hippel PT. New confidence intervals and bias comparisons show that maximum likelihood can beat multiple imputation in small samples. Structural Equation Modeling. 2016;23(3):422-437. doi:10.1080/10705511.2015.1047931

35. Van Buuren S. Flexible Imputation of Missing Data. Boca Raton: CRC press; 2018.

36. Hajek A, König -H-H. Social isolation and loneliness of older adults in times of the CoViD-19 pandemic: can use of online social media sites and video chats assist in mitigating social isolation and loneliness? Gerontology. 2021;67(1):121-124. doi:10.1159/000512793
37. Gorber SC, Tremblay M, Moher D, Gorber B. A comparison of direct vs. self-report measures for assessing height, weight and body mass index: a systematic review. Obesity Rev. 2007;8(4):307-326. doi:10.1111/j.1467-789X.2007.00347.x

38. Egger M, Smith G, Schneider M. Systematic reviews of observational studies. In: Egger M, Smith GD, Altman D, editors. Systematic Reviews in Health Care: Meta-Analysis in Context. 2 ed. London: BMJ Books; 2001:211-227.

Diabetes, Metabolic Syndrome and Obesity: Targets and Therapy

\section{Publish your work in this journal}

Diabetes, Metabolic Syndrome and Obesity: Targets and Therapy is an international, peer-reviewed open-access journal committed to the rapid publication of the latest laboratory and clinical findings in the fields of diabetes, metabolic syndrome and obesity research. Original research, review, case reports, hypothesis formation, expert opinion and commentaries are all considered for publication. The manuscript management system is completely online and includes a very quick and fair peer-review system, which is all easy to use. Visit http://www.dovepress.com/testimonials.php to read real quotes from published authors.

Submit your manuscript here: https://www.dovepress.com/diabetes-metabolic-syndrome-and-obesity-targets-and-therapy-journal 